University of Wollongong

Research Online

Faculty of Business - Papers (Archive)

Faculty of Business and Law

$1-1-2013$

Happiness and job satisfaction in urban China: a comparative study of two generations of migrants and urban locals

Haining Wang

Shandong University, Macquarie University

Zhiming Cheng

University of Wollongong, zhiming@uow.edu.au

Russell Smyth

Monash University

Follow this and additional works at: https://ro.uow.edu.au/buspapers

Part of the Business Commons

Research Online is the open access institutional repository for the University of Wollongong. For further information contact the UOW Library: research-pubs@uow.edu.au 


\title{
Happiness and job satisfaction in urban China: a comparative study of two generations of migrants and urban locals
}

\author{
Abstract \\ This study investigates determinants of happiness and job satisfaction of urban locals, first-generation \\ migrants and new-generation migrants in China's urban workforce. We present evidence to suggest that \\ new-generation migrants are less satisfied with their jobs and lives than first-generation migrants, despite \\ having higher income. This finding is consistent with aspirations rising faster than income in China's fast \\ growing urban economy.
}

\section{Keywords}

study, comparative, china, happiness, job, locals, migrants, urban, generations, satisfaction, two

\section{Disciplines \\ Business}

\section{Publication Details}

Wang, H., Cheng, Z. \& Smyth, R. (2013). Happiness and job satisfaction in urban China: a comparative study of two generations of migrants and urban locals. Monash University. Department of Economics. Discussion Papers, 08/13 1-9. 


\title{
MONASHUniversity
}

Business and Economics

\section{DEPARTMENT OF ECONOMICS}

ISSN 1441-5429

DISCUSSION PAPER 08/13

\section{Happiness and Job Satisfaction in Urban China:}

\section{A Comparative Study of Two Generations of Migrants and Urban Locals}

\author{
Haining Wang', Zhiming Cheng ${ }^{2}$ and Russell Smyth ${ }^{3}$
}

\begin{abstract}
This study investigates determinants of happiness and job satisfaction of urban locals, firstgeneration migrants and new-generation migrants in China's urban workforce. We present evidence to suggest that new-generation migrants are less satisfied with their jobs and lives than firstgeneration migrants, despite having higher income. This finding is consistent with aspirations rising faster than income in China's fast growing urban economy.
\end{abstract}

Keywords: China; Migrants; Subjective wellbeing JEL classification: J28

\footnotetext{
${ }^{1}$ Department of Marketing and Management, Macquarie University

${ }^{2}$ School of Economics, The University of Wollongong

${ }^{3}$ Department of Economics, Monash University
} 


\section{Introduction}

China's rural-urban migration has been described as the largest migration flow in history (Zhao, 1999). Rural-urban migrants have provided the cheap labor that has propelled China's high rate of economic growth for over three decades. Recently, scholars have started to distinguish between first-generation rural-urban migrants, born before 1980, and newgeneration rural-urban migrants, born in 1980 or thereafter.

The new generation accounts for an increasing share of rural-urban migrants as the first generation age and return to the countryside to retire. Compared to first-generation migrants, new-generation migrants are better educated, more socially connected, and they have a stronger tendency towards individualism and consumerism. They also have stronger awareness of their legal and socioeconomic rights, which has resulted in more frequent job changes and workplace unrest. Different from their predecessors for whom return migration was the norm, many new-generation migrants aspire to settle in the cities and to be officially recognized as urban residents with the same rights as urban locals.

There is a growing literature on the determinants of happiness and job satisfaction in transition countries and in China in particular. A subset of this literature examines the determinants of wellbeing of rural-urban migrants (see e.g. Gao \& Smyth, 2011; Knight \& Gunatilaka, 2010; Nielsen et al., 2010). However, each of these studies either focus on firstgeneration migrants or fail to distinguish between first-and-new generation migrants.

In this paper we extend this literature to compare the determinants of happiness and job satisfaction of urban locals, first and new-generation migrants in urban China. We find that, after controlling for other factors potentially correlated with subjective wellbeing, newgeneration migrants have lower levels of happiness and job satisfaction than first-generation migrants. Knight and Gunatilaka (2010) found that happiness of rural-urban migrants in China was lower than both those remaining in rural areas and urban locals. Their explanation was that rural-urban migrants have false expectations about what their migration experience can deliver. Perhaps buoyed by a long period of sustained economic growth, our findings are consistent with the aspirations of new-generation migrants rising faster than income, relative to first-generation migrants, leading to frustration and lower happiness.

\section{Data and model}

The data was collected from 29 provinces and municipalities in mainland China in 2008 through the China General Social Survey (CGSS), which is jointly administrated by Renmin University and Hong Kong University of Science and Technology. There were 5,617 valid responses, among which 2,322 were first-generation migrants, 470 were new-generation migrants, and 2,825 held an urban household registration. Respondent's overall happiness and job satisfaction were measured on a five-point Likert scale ranging from $1=$ very unhappy/dissatisfied to $5=$ very happy/satisfied. The GGSS also collected data on the usual control variables that previous studies suggest are correlated with subjective wellbeing.

Table 1 presents the mean hourly incomes as well as the mean happiness and job satisfaction scores for the three groups. The mean hourly income of new-generation migrants is lower than urban locals, but higher than first-generation migrants. The mean happiness scores of urban locals and new-generation migrants are statistically higher than first-generation migrants and the job satisfaction of urban locals is statistically higher than first-generation and new-generation migrants. However, the mean satisfaction levels in Table 1 do not control for demographic and socioeconomic characteristics correlated with satisfaction. 


\section{[Table 1 here]}

Considering the potential high correlation between the measures of happiness and job satisfaction, we used seemingly unrelated regression, allowing the error terms in the two equations to correlate. The two-equation system is in the form of:

$$
\begin{gathered}
H_{i}=\beta_{0}+\beta_{1} X_{i}+\beta_{2} S_{i}+\mu_{i} \\
J S_{i}=\theta_{0}+\theta_{1} X_{i}+\theta_{2} W_{i}+\varepsilon_{i}
\end{gathered}
$$

where $H$ and $J S$ are happiness and job satisfaction for the $i$ th respondent, respectively; $X$ is a vector of personal characteristics; $S$ is a vector of socioeconomic characteristics; $W$ is a vector of job characteristics; and $\mu$ and $\varepsilon$ are error terms. The two-equation system was estimated simultaneously by applying simulated limited information maximum likelihood, which enabled us to observe the degree of correlation between the observables across equations.

\section{Results}

Table 2 reports the results for the full sample as well as first-generation migrants, newgeneration migrants and urban locals separately. The correlation coefficient for the full sample as well as each of the subsamples is positive and significant. This rejects the null hypothesis that there is no correlation between the error terms, and indicates that unobserved characteristics are positively correlated with happiness and job satisfaction.

The results from the full sample show that, relative to first generation migrants, the happiness and job satisfaction of new-generation migrants is 5.87 per cent and 1.06 per cent lower respectively. The results also suggest that the happiness of urban locals is 5.6 per cent higher than new-generation migrants. Thus, new-generation migrants have the lowest wellbeing among the three cohorts. One explanation is that they have strong aspirations for better lives, career advancement and equal rights, but the opportunities to realize these ambitions are limited. This mismatch results in frustration and lower wellbeing compared with firstgeneration migrants, who have lower expectations and are happier with less.

\section{[Table 2 here]}

The results for the control variables are generally consistent with prior expectations and findings from previous studies. For example, being male, having better health, having larger social networks, being married and being better educated are generally correlated with higher subjective wellbeing. One surprising result was that absolute income is negatively correlated with the happiness of new-generation migrants, albeit only at the 10 per cent level. This may reflect higher income being associated with excessive overtime, high work-related stress and deprivation of legal rights to which new-generation attach importance. ${ }^{4}$

First-generation migrants and urban locals' happiness and urban locals' job satisfaction follow a U-shaped pattern with age, with subjective wellbeing reaching a minimum at around age 40. Having a permanent job significantly increases the job satisfaction of first-generation migrants, indicating that they have a strong desire to avoid job insecurity; but it has no significant effects on new-generation migrants and urban locals. The type of employer has a significant effect on migrants' job satisfaction. Migrants in private enterprises are more

\footnotetext{
${ }^{4}$ Income is potentially endogenous. To address this point, we instrumented for income using parents' years of education. The results were quantitatively similar so we do not report those.
} 
satisfied with their jobs than those in state-owned and collective-owned enterprises, possibly because the private sector discriminates less against migrants and provides a higher income than the state and collective sectors (Démurger et al. 2009). The results of an F-test indicated that there were significant differences in the determinants of happiness between first- and new-generation migrants, and between new-generation migrants and urban residents, but there were no statistically significant differences in the determinants of job satisfaction.

\section{Conclusion}

The composition of China's migrant workforce is changing. New-generation migrants are becoming an increasingly important presence in the Chinese urban landscape. Compared with first-generation migrants, new-generation migrants have higher human and social capital and are more aware of their legal rights. However, in urban China the household registration system continues to underpin labor and social stratification and suppress migrants' socioeconomic rights. Previous research suggests that migrants in urban China have lower subjective wellbeing than urban locals because their expectations are not being fulfilled. Our results suggest that new generation migrants have lower subjective wellbeing than both firstgeneration migrants and urban locals. A likely explanation is that new-generation migrants have higher aspirations than those migrants who preceded them and that these aspirations are not being realized, resulting in relatively lower levels of happiness and job satisfaction. 


\section{References}

Démurger, S., M. Gurgand, S. Li \& X. Yue (2009) Migrants as second-class workers in urban China? A decomposition analysis. Journal of Comparative Economics, 37, 610-628.

Gao, W. and Smyth, R. (2011) What keeps China's migrant workers going? Expectations and happiness among China's floating population. Journal of the Asia Pacific Economy, $16,163-182$.

Knight, J. and Gunatilaka, R. (2010) Great expectations? The subjective wellbeing or ruralurban migrants in China. World Development, 38, 114-124.

Nielsen, I., R. Smyth \& Q. Zhai (2010) Subjective well-being of China's off-farm migrants. Journal of Happiness Studies, 11, 315-333.

Zhao, Y. (1999) Leaving the countryside: Rural to urban migration decisions in China. American Economic Review 89, 281-286. 
Table 1 Summary statistics for happiness, job satisfaction and hourly income

\begin{tabular}{llccccc}
\hline & \multicolumn{2}{c}{ Happiness } & \multicolumn{2}{c}{ Job satisfaction } & \multicolumn{2}{c}{ Hourly income (RMB) } \\
\cline { 2 - 7 } & Mean & SE & Mean & SE & Mean & SE \\
\hline First-generation migrants & 3.5663 & 1.0114 & 3.2821 & 0.7785 & 3.4538 & 0.1788 \\
New-generation migrants & 3.7830 & 0.9596 & 3.2786 & 0.7832 & 5.2075 & 0.6016 \\
Urban locals & 3.7989 & 0.9352 & 3.3914 & 0.7671 & 9.1542 & 0.4037 \\
& A: $\mathrm{t}=-4.2711, \mathrm{p}=0.0000$ & $\mathrm{t}=0.0723, \mathrm{p}=0.9424$ & $\mathrm{t}=-3.6394, \mathrm{p}=0.0003$ \\
t-test for mean comparison & $\mathrm{B}: \mathrm{t}=-0.3413, \mathrm{p}=0.7329$ & $\mathrm{t}=-2.5415, \mathrm{p}=0.0111$ & $\mathrm{t}=-3.8365, \mathrm{p}=0.0001$ \\
& $\mathrm{C}: \mathrm{t}=-8.5583, \mathrm{p}=0.0000$ & $\mathrm{t}=-3.8811, \mathrm{p}=0.0001$ & $\mathrm{t}=-11.9979, \mathrm{p}=0.000$ \\
\hline
\end{tabular}

Notes: A: between first-generation and new-generation migrants; B: between new-generation migrants and urban locals; C: between first-generation migrants and urban locals. 
Table 2 Ordered probit estimates for happiness and job satisfaction (marginal effects)

\begin{tabular}{|c|c|c|c|c|c|c|c|c|}
\hline & \multicolumn{2}{|c|}{ Full sample } & \multicolumn{2}{|c|}{ First-generation migrants } & \multicolumn{2}{|c|}{ New-generation migrants } & \multicolumn{2}{|c|}{ Urban locals } \\
\hline & Happiness & $\begin{array}{c}\text { Job } \\
\text { satisfaction } \\
\end{array}$ & Happiness & $\begin{array}{c}\text { Job } \\
\text { satisfaction }\end{array}$ & Happiness & $\begin{array}{c}\text { Job } \\
\text { satisfaction }\end{array}$ & Happiness & $\begin{array}{c}\text { Job } \\
\text { satisfaction } \\
\end{array}$ \\
\hline \multicolumn{9}{|c|}{ Groups (ref: new-generation migrants) } \\
\hline $\begin{array}{l}\text { First-generation } \\
\text { migrants }\end{array}$ & $\begin{array}{l}0.0587 * * * \\
(0.0197)\end{array}$ & $\begin{array}{l}0.0106^{*} \\
(0.0060)\end{array}$ & & & & & & \\
\hline Urban locals & $\begin{array}{l}0.0560 * * * \\
(0.0179)\end{array}$ & $\begin{array}{c}0.0027 \\
(0.0050)\end{array}$ & & & & & & \\
\hline Gender $($ Male $=1)$ & $\begin{array}{l}-0.0485^{* * *} \\
(0.0081)\end{array}$ & $\begin{array}{l}-0.0057 * * \\
(0.0023)\end{array}$ & $\begin{array}{l}-0.0339 * * * \\
(0.0118)\end{array}$ & $\begin{array}{l}-0.0101^{*} \\
(0.0057)\end{array}$ & $\begin{array}{l}-0.0263 \\
(0.0304)\end{array}$ & $\begin{array}{c}0.0006 \\
(0.0081)\end{array}$ & $\begin{array}{l}-0.0651 * * * \\
(0.0121)\end{array}$ & $\begin{array}{l}-0.0055^{* *} \\
(0.0028)\end{array}$ \\
\hline Age & $\begin{array}{l}-0.0241 * * * \\
(0.0030)\end{array}$ & $\begin{array}{l}-0.0026^{* * *} \\
(0.0009)\end{array}$ & $\begin{array}{l}-0.0107 * \\
(0.0056)\end{array}$ & $\begin{array}{l}-0.0006 \\
(0.0025)\end{array}$ & $\begin{array}{c}0.0123 \\
(0.0824)\end{array}$ & $\begin{array}{c}0.0060 \\
(0.0235)\end{array}$ & $\begin{array}{l}-0.0293 * * * \\
(0.0041)\end{array}$ & $\begin{array}{l}-0.0025^{* *} \\
(0.0010)\end{array}$ \\
\hline $\operatorname{Age}^{2}$ & $\begin{array}{l}0.0003 * * * \\
(0.0000)\end{array}$ & $\begin{array}{l}0.0000 * * * \\
(0.0000)\end{array}$ & $\begin{array}{l}0.0001^{* *} \\
(0.0001)\end{array}$ & $\begin{array}{c}0.0000 \\
(0.0000)\end{array}$ & $\begin{array}{l}-0.0006 \\
(0.0018)\end{array}$ & $\begin{array}{l}-0.0001 \\
(0.0005)\end{array}$ & $\begin{array}{l}0.0004 * * * \\
(0.0001)\end{array}$ & $\begin{array}{l}0.0000 * * * \\
(0.0000)\end{array}$ \\
\hline Years of education & $\begin{array}{l}0.0106^{* * * *} \\
(0.0013)\end{array}$ & $\begin{array}{l}0.0015^{* * * *} \\
(0.0004)\end{array}$ & $\begin{array}{l}0.0083^{* * * *} \\
(0.0018)\end{array}$ & $\begin{array}{c}0.0001 \\
(0.0009)\end{array}$ & $\begin{array}{l}0.0164 * * * \\
(0.0051)\end{array}$ & $\begin{array}{c}0.0010 \\
(0.0015)\end{array}$ & $\begin{array}{l}0.0117 * * * \\
(0.0020)\end{array}$ & $\begin{array}{l}0.0024 * * * \\
(0.0005)\end{array}$ \\
\hline \multicolumn{9}{|c|}{ Marital status (ref: married) } \\
\hline Single & $\begin{array}{l}-0.0672 * * * \\
(0.0142)\end{array}$ & $\begin{array}{l}-0.0082 * * \\
(0.0036)\end{array}$ & $\begin{array}{l}-0.1051 * * * \\
(0.0204)\end{array}$ & $\begin{array}{l}-0.0041 \\
(0.0128)\end{array}$ & $\begin{array}{l}-0.0880 * * \\
(0.0433)\end{array}$ & $\begin{array}{l}-0.0290 * * \\
(0.0136)\end{array}$ & $\begin{array}{l}-0.0787 * * * \\
(0.0212)\end{array}$ & $\begin{array}{l}-0.0024 \\
(0.0055)\end{array}$ \\
\hline Divorced & $\begin{array}{l}-0.0866 * * * \\
(0.0135)\end{array}$ & $\begin{array}{c}0.0043 \\
(0.0061)\end{array}$ & $\begin{array}{l}-0.0620 * * * \\
(0.0206)\end{array}$ & $\begin{array}{c}0.0421 \\
(0.0295)\end{array}$ & $\begin{array}{l}-0.1834 * * * \\
(0.0345)\end{array}$ & $\begin{array}{c}0.0051 \\
(0.0529)\end{array}$ & $\begin{array}{l}-0.1049 * * * \\
(0.0184)\end{array}$ & $\begin{array}{l}-0.0004 \\
(0.0060)\end{array}$ \\
\hline Health status & $\begin{array}{l}0.0642 * * * \\
(0.0042)\end{array}$ & $\begin{array}{l}0.0056^{* * * *} \\
(0.0013)\end{array}$ & $\begin{array}{l}0.0467 * * * \\
(0.0054)\end{array}$ & $\begin{array}{c}0.0047^{*} \\
(0.0026)\end{array}$ & $\begin{array}{l}0.0905 * * * \\
(0.0174)\end{array}$ & $\begin{array}{c}0.0069 \\
(0.0047)\end{array}$ & $\begin{array}{l}0.0779 * * * \\
(0.0069)\end{array}$ & $\begin{array}{l}0.0064 * * * \\
(0.0017)\end{array}$ \\
\hline $\begin{array}{l}\text { Having child(ren) } \\
(\text { Yes }=1)\end{array}$ & $\begin{array}{l}-0.0028 \\
(0.0051)\end{array}$ & $\begin{array}{c}0.0028 * \\
(0.0017)\end{array}$ & $\begin{array}{l}-0.0060 \\
(0.0059)\end{array}$ & $\begin{array}{l}-0.0007 \\
(0.0029)\end{array}$ & $\begin{array}{l}-0.0020 \\
(0.0247)\end{array}$ & $\begin{array}{l}-0.0046 \\
(0.0061)\end{array}$ & $\begin{array}{c}0.0034 \\
(0.0096)\end{array}$ & $\begin{array}{l}0.0069^{* * *} \\
(0.0024)\end{array}$ \\
\hline Hourly income & $\begin{array}{l}0.0008 * * * \\
(0.0003)\end{array}$ & $\begin{array}{l}0.0003 * * * \\
(0.0001)\end{array}$ & $\begin{array}{l}0.0018 * * \\
(0.0008)\end{array}$ & $\begin{array}{l}0.0006^{* *} \\
(0.0002)\end{array}$ & $\begin{array}{l}-0.0020^{*} \\
(0.0012)\end{array}$ & $\begin{array}{c}0.0004 \\
(0.0003)\end{array}$ & $\begin{array}{l}0.0010 * * * \\
(0.0004)\end{array}$ & $\begin{array}{l}0.0002 * * * \\
(0.0001)\end{array}$ \\
\hline Region (ref: western) & & & & & & & & \\
\hline
\end{tabular}




\begin{tabular}{|c|c|c|c|c|c|c|c|c|}
\hline & \multicolumn{2}{|c|}{ Full sample } & \multicolumn{2}{|c|}{ First-generation migrants } & \multicolumn{2}{|c|}{ New-generation migrants } & \multicolumn{2}{|c|}{ Urban locals } \\
\hline & Happiness & $\begin{array}{c}\text { Job } \\
\text { satisfaction }\end{array}$ & Happiness & $\begin{array}{c}\text { Job } \\
\text { satisfaction }\end{array}$ & Happiness & $\begin{array}{c}\text { Job } \\
\text { satisfaction }\end{array}$ & Happiness & $\begin{array}{c}\text { Job } \\
\text { satisfaction }\end{array}$ \\
\hline Eastern & $\begin{array}{l}0.0295^{* * *} \\
(0.0105)\end{array}$ & $\begin{array}{l}0.0081 * * * \\
(0.0031)\end{array}$ & $\begin{array}{c}0.0201 \\
(0.0160)\end{array}$ & $\begin{array}{c}0.0022 \\
(0.0068)\end{array}$ & $\begin{array}{l}0.0791 * * \\
(0.0404)\end{array}$ & $\begin{array}{c}0.0022 \\
(0.0104)\end{array}$ & $\begin{array}{l}0.0330 * * \\
(0.0152)\end{array}$ & $\begin{array}{l}0.0118 * * * \\
(0.0038)\end{array}$ \\
\hline Central & $\begin{array}{c}0.0106 \\
(0.0102)\end{array}$ & $\begin{array}{l}0.0135^{* * *} \\
(0.0037)\end{array}$ & $\begin{array}{l}-0.0066 \\
(0.0130)\end{array}$ & $\begin{array}{c}0.0033 \\
(0.0066)\end{array}$ & $\begin{array}{c}0.0551 \\
(0.0392)\end{array}$ & $\begin{array}{c}0.0040 \\
(0.0107)\end{array}$ & $\begin{array}{c}0.0262 \\
(0.0171)\end{array}$ & $\begin{array}{l}0.0204 * * * \\
(0.0053)\end{array}$ \\
\hline Living space & $\begin{array}{l}0.0003 * * * \\
(0.0001)\end{array}$ & & $\begin{array}{c}0.0002 \\
(0.0001)\end{array}$ & & $\begin{array}{l}0.0011 * * * \\
(0.0004)\end{array}$ & & $\begin{array}{l}0.0005 * * \\
(0.0002)\end{array}$ & \\
\hline Social network & $\begin{array}{l}0.0014 * * * \\
(0.0002)\end{array}$ & & $\begin{array}{l}0.0018 * * * \\
(0.0003)\end{array}$ & & $\begin{array}{l}0.0013 * * * \\
(0.0005)\end{array}$ & & $\begin{array}{l}0.0010 * * * \\
(0.0003)\end{array}$ & \\
\hline Change of social class & $\begin{array}{l}-0.0252 * * * \\
(0.0023)\end{array}$ & & $\begin{array}{l}-0.0246 * * * \\
(0.0032)\end{array}$ & & $\begin{array}{l}-0.0076 \\
(0.0084)\end{array}$ & & $\begin{array}{l}-0.0276^{* * *} \\
(0.0036)\end{array}$ & \\
\hline $\begin{array}{l}\text { Trade union member } \\
(\text { Yes }=1)\end{array}$ & & $\begin{array}{l}-0.0009 \\
(0.0027)\end{array}$ & & $\begin{array}{c}0.0001 \\
(0.0083)\end{array}$ & & $\begin{array}{l}-0.0111 \\
(0.0116)\end{array}$ & & $\begin{array}{l}-0.0006 \\
(0.0031)\end{array}$ \\
\hline \multicolumn{9}{|c|}{ Employer (ref: private enterprise) } \\
\hline SOE & & $\begin{array}{l}0.0059 * * \\
(0.0029)\end{array}$ & & $\begin{array}{c}0.0127 * \\
(0.0070)\end{array}$ & & $\begin{array}{c}0.0115 \\
(0.0123)\end{array}$ & & $\begin{array}{c}0.0051 \\
(0.0035)\end{array}$ \\
\hline $\mathrm{COE}$ & & $\begin{array}{c}0.0012 \\
(0.0038)\end{array}$ & & $\begin{array}{l}-0.0024 \\
(0.0077)\end{array}$ & & $\begin{array}{l}-0.0235 \\
(0.0168)\end{array}$ & & $\begin{array}{c}0.0055 \\
(0.0048)\end{array}$ \\
\hline Others & & $\begin{array}{l}-0.0031 \\
(0.0050)\end{array}$ & & $\begin{array}{c}0.0014 \\
(0.0081)\end{array}$ & & $\begin{array}{c}0.0115 \\
(0.0184)\end{array}$ & & $\begin{array}{l}-0.0075 \\
(0.0074)\end{array}$ \\
\hline White-collar & & $\begin{array}{c}0.0011 \\
(0.0030)\end{array}$ & & $\begin{array}{l}-0.0008 \\
(0.0075)\end{array}$ & & $\begin{array}{l}-0.0003 \\
(0.0136)\end{array}$ & & $\begin{array}{c}0.0008 \\
(0.0034)\end{array}$ \\
\hline $\begin{array}{l}\text { Social insurance } \\
\text { participation }\end{array}$ & & $\begin{array}{l}0.0049 * * * \\
(0.0012)\end{array}$ & & $\begin{array}{c}0.0068 * * \\
(0.0030)\end{array}$ & & $\begin{array}{l}-0.0013 \\
(0.0039)\end{array}$ & & $\begin{array}{l}0.0056^{* * *} \\
(0.0014)\end{array}$ \\
\hline Correlation & $0.2592 * * *$ & & $0.2319 * * *$ & & $0.3667 * * *$ & & $0.2541 * * *$ & \\
\hline
\end{tabular}




\begin{tabular}{|c|c|c|c|c|c|c|c|c|}
\hline & \multicolumn{2}{|c|}{ Full sample } & \multicolumn{2}{|c|}{ First-generation migrants } & \multicolumn{2}{|c|}{ New-generation migrants } & \multicolumn{2}{|c|}{ Urban locals } \\
\hline & Happiness & $\begin{array}{c}\text { Job } \\
\text { satisfaction }\end{array}$ & Happiness & $\begin{array}{c}\text { Job } \\
\text { satisfaction }\end{array}$ & Happiness & $\begin{array}{c}\text { Job } \\
\text { satisfaction }\end{array}$ & Happiness & $\begin{array}{c}\text { Job } \\
\text { satisfaction }\end{array}$ \\
\hline coefficient & $(0.0211)$ & & $(0.0450)$ & & $(0.0724)$ & & $(0.0253)$ & \\
\hline Log likelihood & -10335.7020 & & -3699.9106 & & -838.5512 & & -5731.1109 & \\
\hline No. obs. & 5395 & & 2213 & & 444 & & 2738 & \\
\hline
\end{tabular}

Notes: $1 . * \mathrm{p}<0.1, * * \mathrm{P}<0.05, * * * \mathrm{P}<0.01$

2. Standard errors in parentheses;

3. Health status was self-measured by a five-point scale from 1 (very unhealthy) to 5 (very healthy);

4. Social network was self-measured by calculating the number of friends;

5. Social class was self-measured on a ten-point scale from 1 (highest) to 10 (lowest);

6. Social insurance participation was measured by the number of social insurance schemes in which the respondent participated. 